\title{
MicroRNAs in diabetic cardiomyopathy and clinical perspectives
}

\author{
Qiulian Zhou ${ }^{1,2+}$, Dongchao Lv ${ }^{1,2+}$, Ping Chen ${ }^{1,2+}$, Tianzhao Xu ${ }^{1,2}$, Siyi Fu ${ }^{1,2}$, Jin $\mathrm{Li}^{1,2}$ and Yihua Bei ${ }^{1,2,3 *}$ \\ ${ }^{1}$ Regeneration Lab and Experimental Center of Life Sciences, School of Life Science, Shanghai University, Shanghai, China \\ ${ }^{2}$ Shanghai Key Laboratory of Bio-Energy Crops, School of Life Science, Shanghai University, Shanghai, China \\ ${ }^{3}$ Innovative Drug Research Center of Shanghai University, Shanghai, China \\ *Correspondence: beiyh36@gmail.com \\ ${ }^{\dagger}$ These authors have contributed equally to this work.
}

\section{Edited by:}

Joost Sluijter, University Medical Center Utrecht, Netherlands

Reviewed by:

Jiahong Xu, Shanghai Tongji University, China

Keywords: microRNAs, diabetes, cardiomyopathy, heart failure, biomarker

Diabetes is a progressive metabolic disorder that can ultimately lead to serious chronic vascular complications including renal failure, vision loss, and cardiac dysfunction (Ruiz and Chakrabarti, 2013). Diabetic cardiomyopathy is responsible for higher incidence of sudden cardiac death and represents the leading cause of morbidity and mortality among the diabetic patients (Aksnes et al., 2007; Chavali et al., 2013). Previous studies have indicated that oxidative stress and mitochondrial dysfunction were critically involved in the etiology of diabetes-induced cardiac dysfunction (Sugamura and Keaney, 2011; Styskal et al., 2012), that could subsequently induce a cascade of complex pathophysiological events characterized by early impairments of diastolic function, development of cardiomyocyte hypertrophy, myocardial fibrosis and cardiomyocyte apoptosis, eventually leading to heart failure (Huynh et al., 2014). However, the underlying mechanisms of diabetic cardiomyopathy are far from understood and current therapeutic strategies do not specifically aim at diabetic cardiomyopathy and diabetes-induced heart failure.

MicroRNAs (miRNAs, miRs), a novel class of non-coding RNAs of 22 24 nucleotides in length, act as posttranscriptional regulators of gene expression by binding to the $3^{\prime}$-untranslated region ( $3^{\prime}$-UTR) of target mRNA that induces mRNA degradation and/or translational repression (Lim et al., 2005; Van Rooij, 2011). Given that miRNAs are crucially involved in many critical biological processes including cell proliferation, apoptosis, necrosis, migration and differentiation (Bartel, 2004), desregulated miRNAs contribute to many human diseases including diabetes (Tyagi et al., 2011; Shantikumar et al., 2012; McClelland and Kantharidis, 2014) and cardiovascular diseases (Xiao et al., 2012; Fu et al., 2013; Vickers et al., 2014). Recent studies demonstrate that aberrant expression of miRNAs also participates in the pathogenetic processes mediating diabetic cardiomyopathy, where miR-1, $-133,-141,-206,-223$ have been reported upregulated, whereas miR-133a, -373, and -499 downregulated (Shen et al., 2011; Shantikumar et al., 2012; Asrih and Steffens, 2013). Thus, it is of crucial importance to gain insight into the role of miRNAs in the development of diabetic cardiomyopathy which will help clarify the molecular mechanisms as well as identify novel therapeutic strategies for diabetic cardiomyopathy.

Cardiomyocyte hypertrophy, myocardial fibrosis, and cardiomyocyte apoptosis are important features of diabetic cardiomyopathy (Ruiz and Chakrabarti, 2013). Downregulation of miR-133a induces cardiomyocyte hypertrophy via upregulating the expression of MEF2A and MEF2C, two transcription factors involved in myocardial hypertrophy (Feng et al., 2010). While upregulation of miR-1 and -206 contributes to increased cardiomyocyte apoptosis, via repressing the expression of heat shock protein (Hsp)
60, PIM 1, and IGF-1 receptor (Yu et al., 2008; Shan et al., 2010; Katare et al., 2011). In addition, miR-373 is downregulated in diabetic heart, which is supposed to induce cardiac fibrosis via regulating the expression of p300 (Feng et al., 2008; Chen et al., 2010; Shen et al., 2011; Chavali et al., 2014). Thus, these reports indicate the critical contribution of miRNAs in cardiomyocyte hypertrophy, myocardial fibrosis, and cardiomyocyte apoptosis during the development of diabetic cardiomyopathy.

Hyperglycemia, oxidative stress and mitochondrial damage are involved in the etiology of diabetes-induced cardiac dysfunction and diabetic cardiomyopathy (Shantikumar et al., 2012; Asrih and Steffens, 2013; McClelland and Kantharidis, 2014). Previous study has shown that miR-499 and -133a were markedly downregulated in the diabetic cardiomyocytes, while normalization of oxidant/antioxidant level by the treatment of $\mathrm{N}$-acetylcysteine (NAC) restored the impaired expression of these miRNAs, indicating that hyperglycemia-induced downregulation of miR-499 and -133a was oxidative stress dependent (Yildirim et al., 2013). Similarly, miR-373 was downregulated by hyperglycemia-induced oxidative stress in diabetic cardiomyopathy via p38 MAPK pathway (Shen et al., 2011). MiR-141, a critical regulator of the inner mitochondrial phosphate carrier (Slc25a3), has been shown upregulated in diabetic heart, thus leading to the impaired mitochondrial ATP production 
in the pathogenesis of diabetic cardiomyopathy (Baseler et al., 2012). In terms of cardiomyocyte glucose metabolism, miR-223 was shown to be upregulated in the left ventricular biopsies of diabetic patients, which induced Glut4 protein level in cardiomyocytes and contributed to cardiomyocyte glucose uptake in vitro, indicating that overexpression of miR223 might be a compensatory response to restore glucose metabolism in diabetic heart (Lu et al., 2010).

Accumulating evidence has indicated that circulating miRNAs can be used as sensitive biomarkers for certain diseases such as cardiovascular diseases and cancers (Fabbri, 2010; Tijsen et al., 2012; Xu et al., 2012). Despite that diabetes is among the major risk factors for cardiovascular complications, researches investigating circulating miRNAs in diabetic patients are quite limited. Zampetaki et al. reported deregulation of 12 plasma miRNAs (miR-24, -21, -20b, -15a, -126, -191, $-197,-223,-320,-486,-150$, and $-28-3 p$ ) in diabetic subjects, among which miR126 emerged as a predictor of diabetes mellitus (Zampetaki et al., 2010). A separate study identified 7 upregulated serum miRNAs (miR-9, -29a, -30d, -34, -124, $146 \mathrm{a}$, and -375) in newly diagnosed type 2 diabetic patients as compared to susceptible controls (Kong et al., 2011). Another study identified elevation of miR-144, 192, and 29a in the whole blood of diabetic patients (Karolina et al., 2011), whereas no change was found in miR-126 level which was inconsistent with the report published by Zampetaki et al. (2010). This may be explained by different biosamples detected (plasma vs. whole blood) in these two studies (Zampetaki et al., 2010; Karolina et al., 2011). In addition, miR-503 was found to be enriched in the plasma of diabetic patients with critical limb ischemia (Caporali et al., 2011). However, to date, no specific circulating miRNA has been identified in diabetic cardiomyopathy. The diagnostic and predicted value of circulating miRNAs as biomarkers for diabetic cardiomyopathy remains to be further explored.

Taken together, desregulated miRNAs are potentially involved in the etiology and pathogenetic processes of diabetic cardiomyopathy. An in-depth understanding of their functional roles and molecular mechanisms in the development of diabetic cardiomyopathy will provide better prospects to identify sensitive clinical biomarkers and novel therapeutic targets for diabetic cardiomyopathy.

\section{ACKNOWLEDGMENTS}

This work was supported by the grants from Shanghai Undergraduate Training Programs for Innovation and Enterpreneurship (year 2013, to Siyi Fu).

\section{REFERENCES}

Aksnes, T. A., Kjeldsen, S. E., Rostrup, M., Omvik, P., Hua, T. A., and Julius, S. (2007). Impact of new-onset diabetes mellitus on cardiac outcomes in the Valsartan Antihypertensive Long-term Use Evaluation (VALUE) trial population. Hypertension 50, 467-473. doi: 10.1161/ HYPERTENSIONAHA.106.085654

Asrih, M., and Steffens, S. (2013). Emerging role of epigenetics and miRNA in diabetic cardiomyopathy. Cardiovasc. Pathol. 22, 117-125. doi: 10.1016/j.carpath.2012.07.004

Bartel, D. P. (2004). MicroRNAs: genomics, biogenesis, mechanism, and function. Cell 116, 281-297. doi: 10.1016/S0092-8674(04)00045-5

Baseler, W. A., Thapa, D., Jagannathan, R., Dabkowski, E. R., Croston, T. L., and Hollander, J. M. (2012). miR-141 as a regulator of the mitochondrial phosphate carrier (Slc25a3) in the type 1 diabetic heart. Am. J. Physiol. Cell Physiol. 303, C1244-C1251. doi: 10.1152/ajpcell.00137.2012

Caporali, A., Meloni, M., Völlenkle, C., Bonci, D., Sala-Newby, G. B., Addis, R., et al. (2011). Deregulation of microRNA-503 contributes to diabetes mellitus-induced impairment of endothelial function and reparative angiogenesis after limb ischemia. Circulation 123, 282-291. doi: 10.1161/CIRCULATIONAHA.110.952325

Chavali, V., Tyagi, S. C., and Mishra, P. K. (2013). Predictors and prevention of diabetic cardiomyopathy. Diabetes Metab. Syndr. Obes. 6, 151-160. doi: 10.2147/DMSO.S30968

Chavali, V., Tyagi, S. C., and Mishra, P. K. (2014). Differential expression of dicer, miRNAs, and inflammatory markers in diabetic Ins2+/- Akita hearts. Cell Biochem. Biophys. 68, 25-35. doi: 10.1007/s12013-013-9679-4

Chen, S., Feng, B., George, B., Chakrabarti, R., Chen, M., and Chakrabarti, S. (2010). Transcriptional coactivator p300 regulates glucose-induced gene expression in endothelial cells. Am. J. Physiol. Endocrinol. Metab. 298, E127-E137. doi: 10.1152/ajpendo.00432.2009

Fabbri, M. (2010). miRNAs as molecular biomarkers of cancer. Expert Rev. Mol. Diagn. 10, 435-444. doi: 10.1586/erm.10.27

Feng, B., Chen, S., Chiu, J., George, B., and Chakrabarti, S. (2008). Regulation of cardiomyocyte hypertrophy in diabetes at the transcriptional level. Am. J. Physiol. Endocrinol. Metab. 294, E1119-E1126. doi: 10.1152/ajpendo.00029.2008

Feng, B., Chen, S., George, B., Feng, Q., and Chakrabarti, S. (2010). miR133a regulates cardiomyocyte hypertrophy in diabetes. Diabetes Metab. Res. Rev. 26, 40-49. doi: 10.1002/dmrr.1054
Fu, S., Zhuo, R., Yao, M., Zhang, J., Zhou, H., and Xiao, J. (2013). MicroRNA basis of physiological hypertrophy. Front. Genet. 4:253. doi: 10.3389/fgene.2013.00253

Huynh, K., Bernardo, B. C., McMullen, J. R., and Ritchie, R. H. (2014). Diabetic cardiomyopathy: mechanisms and new treatment strategies targeting antioxidant signaling pathways. Pharmacol. Ther. 142, 375-415. doi: 10.1016/j.pharmthera.2014.01.003

Karolina, D. S., Armugam, A., Tavintharan, S., Wong, M. T. K., Lim, S. C., Sum, C. F., et al. (2011). MicroRNA 144 impairs insulin signaling by inhibiting the expression of insulin receptor substrate 1 in type 2 diabetes mellitus. PLOS ONE 6:e22839. doi: 10.1371/journal.pone.0022839

Katare, R., Caporali, A., Zentilin, L., Avolio, E., SalaNewby, G., Oikawa, A., et al. (2011). Intravenous gene therapy with PIM-1 via a cardiotropic viral vector halts the progression of diabetic cardiomyopathy through promotion of prosurvival signaling. Circ. Res. 108, 1238-1251. doi: 10.1161/CIRCRESAHA.110.239111

Kong, L., Zhu, J., Han, W., Jiang, X., Xu, M., Zhao, Y., et al. (2011). Significance of serum microRNAs in pre-diabetes and newly diagnosed type 2 diabetes: a clinical study. Acta Diabetol. 48, 61-69. doi: 10.1007/s00592-010-0226-0

Lim, L. P., Lau, N. C., Garrett-Engele, P., Grimson, A., Schelter, J. M., Castle, J., et al. (2005). Microarray analysis shows that some microRNAs downregulate large numbers of target mRNAs. Nature 433, 769-773. doi: 10.1038/nature03315

Lu, H., Buchan, R. J., and Cook, S. A. (2010). MicroRNA-223 regulates Glut4 expression and cardiomyocyte glucose metabolism. Cardiovasc. Res. 86, 410-420. doi: 10.1093/cvr/cvq010

McClelland, A. D., and Kantharidis, P. (2014). microRNA in the development of diabetic complications. Clin. Sci. Lond. Engl. 126, 95-110. doi: 10.1042/CS20130079

Ruiz, M. A., and Chakrabarti, S. (2013). MicroRNAs: the underlying mediators of pathogenetic processes in vascular complications of diabetes. Can. J. Diabetes 37, 339-344. doi: 10.1016/j.jcjd.2013.07.003

Shan, Z.-X., Lin, Q.-X., Deng, C.-Y., Zhu, J.-N., Mai, L.-P., Liu, J.-L., et al. (2010). miR-1/miR-206 regulate Hsp60 expression contributing to glucosemediated apoptosis in cardiomyocytes. FEBS Lett. 584, 3592-3600. doi: 10.1016/j.febslet.2010.07.027

Shantikumar, S., Caporali, A., and Emanueli, C. (2012). Role of microRNAs in diabetes and its cardiovascular complications. Cardiovasc. Res. 93, 583-593. doi: 10.1093/cvr/cvr300

Shen, E., Diao, X., Wang, X., Chen, R., and $\mathrm{Hu}$, B. (2011). MicroRNAs involved in the mitogen-activated protein kinase cascades pathway during glucose-induced cardiomyocyte hypertrophy. Am. J. Pathol. 179, 639-650. doi 10.1016/j.ajpath.2011.04.034

Styskal, J., Van Remmen, H., Richardson, A., and Salmon, A. B. (2012). Oxidative stress and diabetes: what can we learn about insulin resistance from antioxidant mutant mouse models? Free Radic. Biol. Med. 52, 46-58. doi: 10.1016/j.freeradbiomed.2011.10.441

Sugamura, K., and Keaney, J. F. Jr. (2011). Reactive oxygen species in cardiovascular 
disease. Free Radic. Biol. Med. 51, 978-992. doi: 10.1016/j.freeradbiomed.2011.05.004

Tijsen, A. J., Pinto, Y. M., and Creemers, E. E. (2012). Circulating microRNAs as diagnostic biomarkers for cardiovascular diseases. Am. J. Physiol. Heart Circ. Physiol. 303, H1085-H1095. doi: 10.1152/ajpheart.00191.2012

Tyagi, A. C., Sen, U., and Mishra, P. K. (2011). Synergy of microRNA and stem cell: a novel therapeutic approach for diabetes mellitus and cardiovascular diseases. Curr. Diabetes Rev. 7, 367-376. doi: 10.2174/157339911797579179

Van Rooij, E. (2011). The art of microRNA research. Circ. Res. 108, 219-234. doi: 10.1161/CIRCRESAHA.110.227496

Vickers, K. C., Rye, K.-A., and Tabet, F. (2014). MicroRNAs in the onset and development of cardiovascular disease. Clin. Sci. Lond. Engl. 126, 183-194. doi: 10.1042/CS20130203

Xiao, J., Liang, D., Zhang, H., Liu, Y., Zhang, D., Liu, Y., et al. (2012). MicroRNA-204 is required for differentiation of human-derived cardiomyocyte progenitor cells. J. Mol. Cell. Cardiol. 53, 751-759. doi: 10.1016/j.yjmcc.2012.08.024
Xu, J., Zhao, J., Evan, G., Xiao, C., Cheng, Y., and Xiao, J. (2012). Circulating microRNAs: novel biomarkers for cardiovascular diseases. J. Mol. Med. Berl. Ger. 90, 865-875. doi: 10.1007/s00109-0110840-5

Yildirim, S. S., Akman, D., Catalucci, D., and Turan, B. (2013). Relationship between downregulation of miRNAs and increase of oxidative stress in the development of diabetic cardiac dysfunction: junctin as a target protein of miR-1. Cell Biochem. Biophys. 67, 1397-1408. doi: 10.1007/s12013-0139672-y

Yu, X.-Y., Song, Y.-H., Geng, Y.-J., Lin, Q.-X., Shan, Z.-X., Lin, S.-G., et al. (2008). Glucose induces apoptosis of cardiomyocytes via microRNA-1 and IGF-1. Biochem. Biophys. Res. Commun. 376, 548-552. doi: 10.1016/j.bbrc.2008.09.025

Zampetaki, A., Kiechl, S., Drozdov, I., Willeit, P., Mayr, U., Prokopi, M., et al. (2010). Plasma microRNA profiling reveals loss of endothelial miR-126 and other microRNAs in type 2 diabetes. Circ. Res. 107, 810-817. doi: 10.1161/CIRCRESAHA.110. 226357
Conflict of Interest Statement: The authors declare that the research was conducted in the absence of any commercial or financial relationships that could be construed as a potential conflict of interest.

Received: 12 May 2014; accepted: 30 May 2014; published online: 25 June 2014.

Citation: Zhou Q, Lv D, Chen P, Xu T, Fu S, Li J and Bei $Y$ (2014) MicroRNAs in diabetic cardiomyopathy and clinical perspectives. Front. Genet. 5:185. doi: 10.3389/ fgene.2014.00185

This article was submitted to Epigenomics and Epigenetics, a section of the journal Frontiers in Genetics.

Copyright (C) 2014 Zhou, Lv, Chen, Xu, Fu, Li and Bei. This is an open-access article distributed under the terms of the Creative Commons Attribution License (CC BY). The use, distribution or reproduction in other forums is permitted, provided the original author(s) or licensor are credited and that the original publication in this journal is cited, in accordance with accepted academic practice. No use, distribution or reproduction is permitted which does not comply with these terms. 